\title{
Electrochemical characterization of clean shape-controlled Pt nanoparticles prepared in presence of oleylamine/oleic acid
}

\author{
Rosa M. Arán-Ais ${ }^{a}$, Francisco J. Vidal-Iglesias $^{a}$, Jose Solla-Gullón $^{a}$, Enrique Herrero ${ }^{a}$, Juan. M. Feliu $_{*}^{a}$ \\ a Instituto de Electroquímica, Universidad de Alicante Ap. 99, E-03080, Alicante, Spain \\ * e-mail: juan.feliu@ua.es
}

Received: ((will be filled in by the editorial sttaff))

Accepted: ((will be filled in by the editorial sttaff))

\begin{abstract}
A collection of shape-controlled Pt nanoparticles has been prepared using two different and previously described methodologies, both using oleylamine/oleic acid as capping material/solvent. A new decontamination protocol is presented to effectively clean the surface of the different nanoparticles thus allowing a full exposure of their surface area and consequently to make the most of their surface structure dependent reactivity. Subsequently, the clean shapecontrolled Pt nanoparticles have been electrochemically characterized and their electrocatalytic properties evaluated towards some surface structure reactions of interest. The results indicate that the full characterization of the surface structure cannot be done exclusively by the available microscopy techniques, since it is very difficult to determine the presence of surface defects. Additional surface characterization probes, such as those provide by electrochemical surface sensitive reactions have been used to assess the surface structure of the samples.
\end{abstract}

Keywords: Platinum, Nanoparticles, Shapes, Cleaning, Electrocatalysis

DOI: 10.1002/elan.((will be filled in by the editorial sttaff))

\section{Introduction}

In the last years, the synthesis and electrochemical properties of shape-controlled $\mathrm{Pt}$ and $\mathrm{Pt}$ alloy nanoparticles have been widely explored with the main objective of obtaining catalysts that are more efficient. ${ }^{[1]}$ Thus, the use of such nanomaterials has clearly shown that both the reactivity and selectivity of the nanoparticles can be modulated by controlling their morphology, because the surface structure of the nanoparticles may contain very different reactive surface sites depending on their shape. In addition, these systems are ideal candidates to obtain the experimental correlations using single-crystal electrodes as model probes.

In general, the preparation of these shapecontrolled nanoparticles requires the use of surfacestabilizing agents which adsorb during the nucleation and growth of the nanoparticles, inducing the synthesis of nanoparticles with a preferential shape and consequently, to the formation of nanoparticles with a particular surface structure. However, it is well established from the knowledge obtained in previous single crystal studies that a full understanding of the correlations between surface structure and surface reactivity is only possible with clean surfaces. Consequently, it is of fundamental importance to develop efficient cleaning procedures to remove the specific stabilizing agents employed during the synthesis. In addition, and this is not a trivial point, this removal of reagents must be performed without damaging or changing their surface properties. Thus, for instance, neither the electrochemical activation procedure nor ozone cleaning should be employed if the surface properties should be preserved because of the wellestablished surface modification that takes place during these treatments. ${ }^{[2]}$ At this respect, it is worth noting that the shape of a nanoparticle is just an indication of its possible surface structure, i.e. its surface atomic arrangement. It is very important to differentiate between shape and surface structure at an atomic level, because surface defects are not detectable under standard microscopy techniques. For example, cubic platinum nanoparticles treated under UV/ozone radiation (used as cleaning procedure) showed an unperturbed particle size and shape with respect to that obtained prior to the treatment but different reactivity and selectivity, due to the presence of a high number of defects on the nanoparticle surface. ${ }^{[2]}$ These defects were created during the cleaning procedure. In this way, the shape and the surface structure of the nanoparticles must be
Con formato: Español (alfab. internacional)

Con formato: Español (alfab. internacional)

Con formato: Español (alfab. internacional)

Con formato: Español (alfab. internacional)

Con formato: Español (alfab.

internacional) 
independently evaluated, but being the latter, that is, the particular surface atom arrangement, the determining parameter that controls their catalytic or electrocatalytic properties.

As previously stated, the use of clean surfaces is an outstanding requirement in Electrocatalysis. In this sense, the evaluation of the cleanliness as well as the determination of the presence of different symmetry sites on Pt surfaces can be made by visualizing the so-called hydrogen adsorption/desorption region which is a pure in situ electrochemical process. ${ }^{[3]}$ In addition, the adsorption or desorption charges for this reaction are known to be directly proportional to the amount of surface atoms thus allowing the electroactive surface area of the sample to be evaluated.

Over the last few years, many papers have been published dealing with the synthesis of Pt and Pt-based alloy nanoparticles in organic solutions, where a mixture of oleylamine/oleic acid are used as surfactant, solvent and reducing agent depending on other synthesis parameters. ${ }^{[4]}$ This method allows the synthesis of high quality nanocrystals with different sizes and shapes by controlling the ratio between the different components of the synthesis and the temperature. Various methods existing in the literature were applied to remove the oleylamine surfactant, such as washing with organic solvents like hexane and/or ethanol, $\left.{ }^{[4 b}, 4 c\right]$ thermal annealing in air, ${ }^{[5]}$ acetic acid, ${ }^{[5-6]}$ UV-Ozone $^{[5]}$ and plasma treatment, ${ }^{[7]}$ as well as the potential cycling in order to bring additional cleaning to the nanocatalyst. ${ }^{[5,7]}$ These harsh treatment conditions can strongly perturb the surface structure of the nanoparticles, significantly modifying their catalytic properties, but without altering their size and shape. On the other hand, literature in this topic shows voltammetric profiles obtained for $\mathrm{Pt}$ nanoparticles with partially blocked surface and poorly defined adsorption peaks, revealing the presence of impurities due to the incomplete removal of the oleylamine/oleic acid of the surface of the nanoparticles. That means that the decontamination procedure is not enough and that the catalytic or electrocatalytic activity shown by the particles could not be correlated with its surface structure.

To the best of our knowledge, only the method proposed by $\mathrm{H}$. Yang et al. ${ }^{[8]}$ has demonstrated a complete removal of the oleylamine/oleic acid from the surface of the nanoparticles synthesized by this procedure, as shown by their voltammetric results. In addition, this protocol seems to be also effective for removing PVP surfactant. Nevertheless, this cleaning method requires an in-situ electrochemical treatment that has an important disadvantage for practical purposes where larger amounts of nanoparticles are needed.
Consequently, new decontamination procedures should be developed to overcome these limitations.

In this work, we report a novel approach for the preparation of clean shape-controlled Pt nanoparticles prepared using the oleylamine/oleic acid system. In this paper, we will describe the application of different cleaning procedures and will discuss their pronounce impact on their electrocatalytic behavior. Finally, for the clean Pt nanoparticles, a detailed electrochemical characterization as well as some of their electrocatalytic properties will be reported and evaluated in terms of correlation between surface structure and reactivity/selectivity.

\section{Experimental}

The syntheses of the different Pt nanoparticles were performed using similar methodologies to those previously reported by J. Zhang and J. Fang ${ }^{[4 b]}$ and Y. Kang et al. ${ }^{[4]}$ in which oleylamine and oleic acid (OLA/OA) are used as capping agents, metal carbonyls as additives and, in some cases, benzyl ether is used as co-solvent. In particular, spherical and cubic Pt nanoparticles were synthesized as described in the general strategy proposed by J. Zhang and J. Fang. ${ }^{[4]}$ In the present work, these samples will be called as Poly-PtA and (100)-Pt-A, respectively. This synthesis is carried out in the presence of $\mathrm{W}(\mathrm{CO})_{6}$, and the mixture oleylamine and oleic acid act as a pair of solvent/reducing and binding agents. The synthesis of the spherical nanoparticles is exactly the same to that used for the cubic sample but in absence of $\mathrm{W}(\mathrm{CO})_{6}$. On the other hand, spherical, cubic and truncated cubic and octahedral Pt nanoparticles were prepared as detailed in reference ${ }^{[4 c]}$. These samples will be called as Poly-Pt-B, (100)-Pt-B, (100)-(111)-Pt-B and (111)-(100)-Pt-B, respectively. In this case, $\mathrm{Mn}_{2}(\mathrm{CO})_{10}$ is use as additive and the fine control over the shape of the nanoparticles is simply achieved by changing the reaction temperature and/or the amount of $\mathrm{Mn}_{2}(\mathrm{CO})_{10}$ added. Importantly, all these syntheses were performed using benzyl ether as cosolvent.

Once the synthetic reactions are completed, the black dispersion is cooled down to room temperature and the nanoparticles are isolated by centrifugation (6000 rpm, $5 \mathrm{~min}$ ). After discarding the supernatant liquid, the nanoparticles were washed using different treatments. The effectiveness of these cleaning protocols will be discussed in a following section of this paper. In the optimal cleaning, the samples were washed twice with a mixture of hexane and ethanol. The precipitates were then re-dispersed in $20 \mathrm{~mL}$ of methanol and a pellet of $\mathrm{NaOH}$ (about $0.2 \mathrm{~g}$ ) was added to the dispersion, which 
was sonicated for $5 \mathrm{~min}$. After the nanoparticles precipitated, the alkaline solution of methanol was removed and the particles were washed with acetone. This procedure $\mathrm{MeOH}+\mathrm{NaOH} /$ acetone was repeated at least three times, and after that, the nanoparticles were washed twice with ultra pure water. The nanoparticles were finally dispersed in ultra pure water.

Transmission Electron Microscopy (TEM) experiments were performed with a JEOL JEM-2010 microscope working at $200 \mathrm{kV}$ and with a JEOL JEM-1400 Plus working at $120 \mathrm{kV}$. The samples were prepared by placing a drop of the hexanolic solution onto a Formvarcovered copper grid and evaporating it in air at room temperature. For each sample, usually about 200-300 particles from different parts of the grid were used to estimate the mean diameter and size distribution of the nanoparticles.

| The electrochemical characterization of the different Pt nanoparticles was performed at room temperature in 0.5 $\mathrm{M} \mathrm{H}_{2} \mathrm{SO}_{4}$ solution, which was every day prepared from Milli-Q ${ }^{\circledR}$ water and Merck "p.a." sulfuric acid. A threeelectrode electrochemical cell was used with a platinum wire as a counter electrode and a reversible hydrogen electrode (RHE), connected to the cell through a Luggin capillary, as reference electrode. The solutions were deaerated with Argon (Ar N50, Air Liquide) and the electrode potential was controlled using a PGSTAT30 AUTOLAB system and a VMP3 multichannel potentiostat (BioLogic) with an NStat configuration (1 counter electrode, 1 reference electrode and 8 working electrodes working simultaneously). The particles were deposited in a gold collector, which was mechanically polished with alumina and rinsed with ultra-pure water to eliminate the nanoparticles from previous experiments. A droplet of the nanoparticles suspension was pipetted onto the polished Au substrate and the water was allowed to evaporate in an $\mathrm{Ar}$ atmosphere. The determination of the active surface area of the different Pt nanoparticles was determined in $\mathrm{H}_{2} \mathrm{SO}_{4}$ by the charge involved in the socalled hydrogen UPD region assuming $230 \mu \mathrm{C} \mathrm{cm}{ }^{-2}$ for the total charge after the subtraction of the double layer charging contribution as previously discussed. ${ }^{[3]}$ CO oxidation experiments were carried out by bubbling $\mathrm{CO}$ (g) (N47, Air Liquide) through the electrolyte at $0.1 \mathrm{~V}$ until complete blockage of the surface, which was monitored by cycling the electrode between 0.05 and 0.3 $\mathrm{V}$. After that, CO was removed from the solution by bubbling $\mathrm{Ar}$ for at least $20 \mathrm{~min}$ and CO-stripping voltammograms were performed at $20 \mathrm{mV} \mathrm{s}^{-1}$ in order to oxidize the $\mathrm{CO}$ molecules adsorbed on the surface in a single sweep.

The electrochemically determination of the percentage of (111) and (100) surface domains was performed using the adsorption of bismuth (Bi) and germanium (Ge), respectively, as described in previous contributions. ${ }^{[3 \text { a, 9] }}$ In summary, irreversible adsorption of bismuth was performed by spontaneous deposition from a saturated solution of bismuth (III) oxide in $0.5 \mathrm{M}$ sulfuric acid. After deposition, the electrode surface was rinsed with water and immersed in the electrochemical cell. The adsorption of germanium was performed as described previously from $10^{-2} \mathrm{M}$ solutions of $\mathrm{GeO}_{2}$ in $1 \mathrm{M} \mathrm{NaOH}$. The electrode with the droplet attached was immersed in the cell at $0.1 \mathrm{~V}$. Residual contamination of the cell by germanium ions was checked after each experiment.

\section{Results and Discussion}

\subsection{TEM characterization of the different Pt nanoparticles}

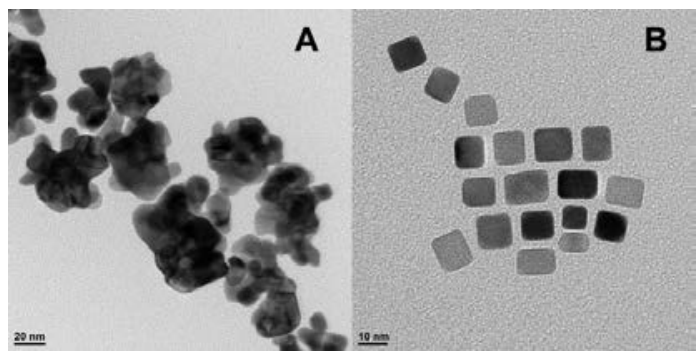

Fig. 1. TEM images of (A) quasi-spherical (Poly-Pt-A) and (B) cubic ((100)-Pt-A) Pt nanoparticles prepared by the method described in ref ${ }^{[\mathbf{a b}]}$.

Figure 1 shows some representative TEM images of the quasi-spherical and cubic Pt nanoparticles prepared using the general strategy proposed by J. Zhang and J. Fang. ${ }^{[4 \mathrm{~b}]}$ The sample prepared in absence of $\mathrm{W}(\mathrm{CO})_{6}$ (Fig. 1A) shows the presence of agglomerated and quasi-spherical Pt nanoparticles, indicating the lack of control over the shape of the sample. In contrast, the sample prepared in presence of the specific additive clearly shows a cubiclike morphology (Fig. 1B) and a particle size about 10.2 $\pm 1.5 \mathrm{~nm}$, in good agreement with the expected size and shape of the samples from literature. ${ }^{[4 b]}$

Figure 2 shows some representative TEM images of the quasi-spherical, cubic and truncated cubic and octahedral Pt nanoparticles prepared using $\mathrm{Mn}_{2}(\mathrm{CO})_{10}$ as additive and benzyl ether as a co-solvent and at different reaction temperatures. ${ }^{[4 c, 10]}$ Quasi-spherical Pt nanoparticles are synthesized in the presence of a high concentration of metal carbonyl, thus yielding in a higher number of nuclei and producing smaller nanoparticles. However, in 
presence of a appropriate amounts of $\mathrm{Mn}_{2}(\mathrm{CO})_{10}$, different morphologies can be clearly observed as a function of the reaction temperature. Nevertheless, it is worth noting that whereas cubic nanoparticles (Figure 2B) are clearly distinguished, the TEM images corresponding to the truncated cubic (Figure 2C) and truncated octahedral (Figure 2D) Pt nanoparticles are less discernible. This is due to the fact that the TEM micrographs only provide a $2 \mathrm{D}$ projection of these particles, and that the main differences between these samples just imply a different degree of truncation. In terms of mean particle size, cubic and truncated cubic and octahedral Pt nanoparticles show a particle size about 10 $\pm 1 \mathrm{~nm}, 10.5 \pm 0.8 \mathrm{~nm}$ and $12 \pm 1 \mathrm{~nm}$ respectively. The particle size of the quasi-spherical ones is about $3.7 \pm 0.4$ $\mathrm{nm}$. As discussed in previous contributions, these quasispherical, cubic and truncated cubic and octahedral Pt nanoparticles can be assigned, in terms of surface structure, to polyoriented, (100), (100)-(111) and (111)(100) preferentially oriented Pt nanoparticles, respectively. ${ }^{[1 \mathrm{a}, 1 \mathrm{c}, 3 \mathrm{~b}, 11]}$

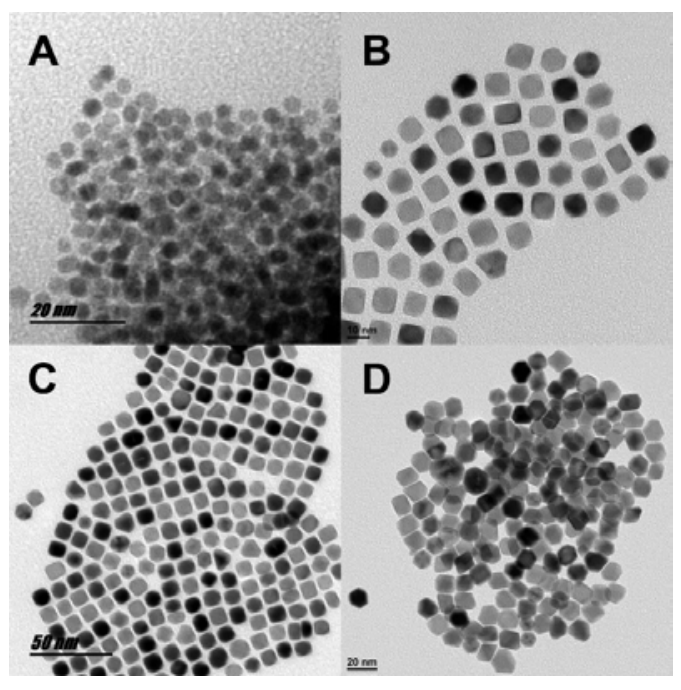

Fig. 2. TEM images of Pt nanoparticles prepared by the method described in ref ${ }^{\text {I4cl: }}$ (A) Polyoriented, (B) (100), (C) (100)(111) and (D) (111)-(100) preferentially oriented.

\subsection{Electrochemical characterization}

\subsubsection{Evaluation of the different cleaning procedures}

As previously stated in the Introduction section of the paper, a full understanding of the correlations between shape/surface structure and surface reactivity is only possible on clean surfaces. At this respect, it is well- established that the so-called hydrogen adsorption/desorption region to be an excellent process to evaluate not only the presence of different active sites but also the level of cleanliness of the samples. Thus, in the following, we will describe in detail the different attempts to eliminate the organic molecules adsorbed on the surface of the Pt nanoparticles, synthesized by the oleylamine/oleic acid method, while keeping unperturbed their morphology and surface structure. In particular, once the nanoparticles were synthesized, the resulting black dispersion was treated following different protocols in an attempt to remove the surfactant from the surface. Initially, we tested similar chemical cleaning procedures to those used in the literature for Pt nanoparticles also obtained in the OLA/OA system, ${ }^{[4 \mathrm{~b}, 4 \mathbf{c}]}$ which involve the use of hexane or ethanol as cleaning agents. In addition, these samples were also treated following a decontamination procedure previously used for $\mathrm{Pt}$ nanoparticles prepared in water-in-oil microemulsion (w/o) that involves a chemical washing with acetone and then with ultrapure water. Figure 3 summarizes the electrochemical response of the samples submitted to these different cleaning protocols. The results obtained clearly indicate that none of the used cleaning protocols is able to effectively remove the capping agents used during the synthesis of the nanomaterials, because the typical peaks associated with hydrogen adsorption on a polycrystalline sample in sulfuric acid solutions are absent. In particular, whereas the use of acetone and ethanol are evidently insufficient and the surface of the samples remains almost inaccessible for the electrochemical reactions, the sample cleaned with hexane shows a hydrogen profile poorly-defined and thus denoting the presence of residual amounts of organic contaminants at the surface of the Pt sample.

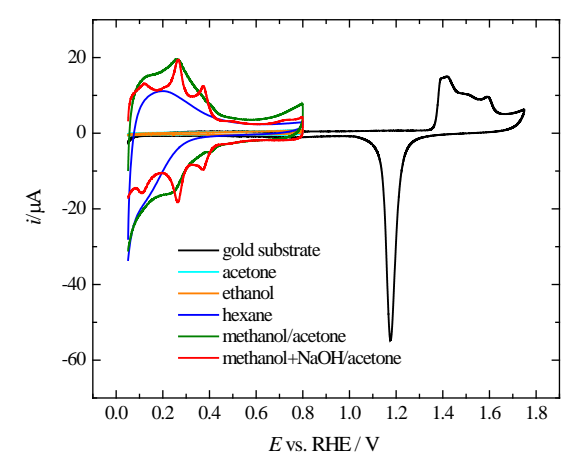

Fig. 3. Voltammetric profiles of (100)-Pt-A nanoparticles supported on a gold substrate. The nanoparticles were cleaned with acetone (cyan line), ethanol (orange line), hexane (blue 


\section{Full Paper}

line), methanol (green line) and methanol enriched with sodium hydroxide/acetone (red line). Test solution: $0.5 \mathrm{M} \mathrm{H}_{2} \mathrm{SO}_{4}$. Sweep rate $=50 \mathrm{mV} \mathrm{s}^{-1}$.

Subsequently, classical CO adsorption-stripping experiments were also performed to evaluate whether $\mathrm{CO}$ molecules are capable of displacing the impurities present at the surface of the nanoparticles. In case of the samples treated with acetone or ethanol, no CO oxidation peak was observed. This result points out that $\mathrm{CO}$ is not able to remove the adsorbed species remaining from the OLA/OA synthesis which remain strongly adsorbed at the platinum surface. However, for that sample cleaned with hexane, the $\mathrm{CO}$ is able adsorb on those clean surface sites. This CO adsorption is marked by the decrease of the observed hydrogen charge. However, the subsequent CO stripping not only takes place without the appearance of a well-defined CO oxidation peak but also is unable to improve its surface cleanness of the sample. Finally, in the cases under discussion, the samples are electrochemically activated by potential cycling between the hydrogen and oxygen evolution regions. Despite a slightly increase of the charge in the hydrogen adsorption/desorption region, the voltammetric profile in the hydrogen region does not show any characteristic feature. In addition, it is well established this electrochemical treatment produces an important perturbation and disordering of the surface structure, which, by itself, prevents its application. ${ }^{[2 \mathrm{a}, 2 \mathrm{~b}]}$

Further essays were performed with methanol interspersed with acetone washes. As previously stated, the use of acetone does not remove the organic ligands from the surface of the nanoparticles. Nevertheless, in this case it is used to dilute the remaining alkaline solution of methanol after the precipitation of the nanoparticles. In addition, the use of acetone is an indispensable step to finally disperse the nanoparticles in water due to its immiscibility with methanol. The resulting Pt nanoparticles were finally dispersed in ultrapure water. The voltammetric profile corresponding to the Pt nanoparticles cleaned with this procedure is also reported in Figure 3. In this case, some interesting features are slightly drawn in the voltammogram, but the absence of sharpness in the voltammetric peaks indicates that the surface of the nanoparticles is not complete clean. $\mathrm{CO}$ adsorption and further oxidation was also tested in this sample, and the results are reported in Figure 4A. After two CO adsorption/stripping processes, the voltammetric profile of the nanoparticles shows defined and symmetric features, which evidence an important improvement of the surface cleanness of the sample. Additional CO adsorption/stripping experiments did not improve the level of cleanliness of the surface of the nanoparticles, in terms of definition and symmetry of the adsorption states. It should be highlighted that clean Pt surfaces should have voltammograms that are perfectly symmetrical with respect to the potential axis. It is also worth noting that the voltammetric profile of the $\mathrm{Pt}$ nanoparticles corresponds to a preferential (100) orientation, i.e. cubic nanoparticles, as can be deduced from the signals at 0.27 and $0.37 \mathrm{~V}$, which are wellknown to be related to (100) step sites and (100) terraces, respectively. This behaviour is in good agreement with the TEM images obtained for this sample. In order to demonstrate that, after this specific cleaning, the surface is fully available for any electrochemical reaction, oxidation/reduction cycles were carried out. Figure 4B shows the evolution of the voltammetric profile of this particular sample after being subjected to a potential cycling. The results obtained indicate that the hydrogen charge which is related to the electroactive surface area of the samples, remain relatively constant for the different potential steps thus suggesting the effective cleanness of the samples. Obviously, the changes in the charge distribution among the different peaks are due to the well-established order disruption of the surface, but not due to an improvement of its cleanliness level. In particular, as discussed in previous works, ${ }^{[2]}$ the contribution associated to the wide (100) domains is particularly affected by the potential cycling and it is decreased at the expense of an increase in the features related with (100) and (110) step sites. This fact again points out that potential cycling, that is, electrochemical activation, should not be used with shaped-controlled $\mathrm{Pt}$ nanoparticles as cleaning protocol to avoid the damage of their particular surface structure.

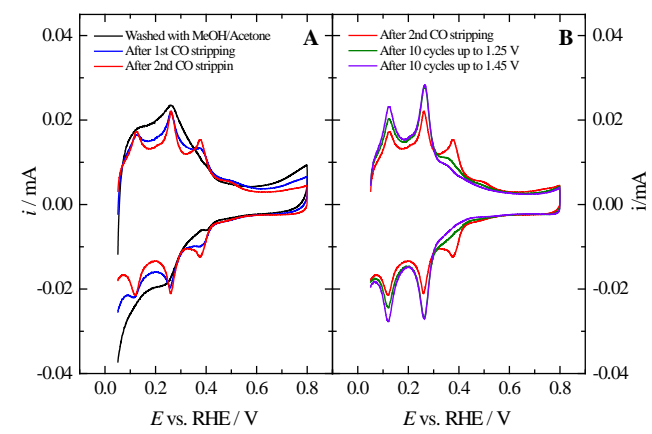

Fig. 4. Voltammetric profiles of (100)-Pt-A nanoparticles washed with MeOH/Acetone. (A) Effect of CO adsorption/oxidation. (B) Effect of electrochemical activation. Test solution: $0.5 \mathrm{M} \mathrm{H}_{2} \mathrm{SO}_{4}$, sweep rate: $50 \mathrm{mV} \mathrm{s}^{-1}$. 
Despite this latter procedure can be considered as a good method to remove the organic compounds remaining from the OLA/OA synthesis, further attempts were carried out in order to obtain a method to clean $\mathrm{Pt}$ nanoparticles without the necessity of the CO adsorption/oxidation steps. This goal was achieved by simply alkalizing the methanol solvent used in the last described procedure. Figures 3 and 5 show the voltammetric response of cubic Pt nanoparticles cleaned following that protocol. Their voltammetric profile clearly shows the features related to the so-called hydrogen adsorption/desorption on the different symmetry sites. As aforementioned, cleanliness is guaranteed by the reversibility and definition of the adsorption desorption states. Moreover, in contrast with the previous protocol, a single $\mathrm{CO}$ adsorption/oxidation treatment (Figure 5) just slightly improves the definition of the hydrogen peaks being the voltammetric responses before and after the $\mathrm{CO}$ treatment almost identical. Consequently, this latter decontamination procedure was extended to all shape-controlled Pt nanoparticles prepared under similar conditions, that is, in the presence of OLA/OA. A detailed analysis of the electrochemical response of the different shaped Pt nanoparticles as well as their electrocatalytic activity towards some particular reaction will be discussed in forthcoming sections of this paper.

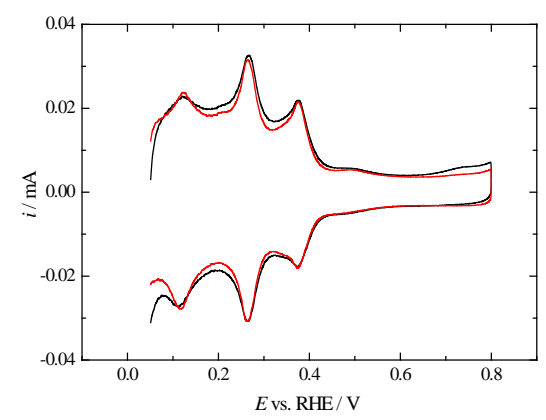

Fig. 5. Cyclic voltammograms of cubic Pt nanoparticles obtained by the procedure in ref ${ }^{[4 b]}$ and washed with Methanol+NaOH/Acetone: before (black line) and after (red line) the $\mathrm{CO}$ adsorption/oxidation treatment. Test solution: 0.5 $\mathrm{M} \mathrm{H}_{2} \mathrm{SO}_{4}$, sweep rate $=50 \mathrm{mV} \mathrm{s}^{-1}$.

\subsubsection{Voltammetric characterization of the nanoparticles}

Figure 6 shows the characteristic voltammetric profiles of the different shaped Pt nanoparticles in $0.5 \mathrm{M} \mathrm{H}_{2} \mathrm{SO}_{4}$, after effective cleaning of their surfaces. In all cases, the sharpness, good definition and the symmetry of the adsorption states are clear proofs of their effective surface cleanliness. In previous contributions we already reported a detailed analysis of the main characteristic voltammetric features observed in this media with shaped Pt nanoparticles prepared using other synthetic routes. ${ }^{[3]}$ Very briefly, the main voltammetric features include i) the peak at $0.125 \mathrm{~V}$, which is related to (110)-type sites, ii) the peak at $0.27 \mathrm{~V}$ which contains two contributions from (100) step sites on (111) terraces and the sites close to the steps on the (100) terraces, iii) the signals 0.35 $0.37 \mathrm{~V}$ attributed to (100) bidimensional terraces and iv) the signal at about $0.5 \mathrm{~V}$, related to the bidimensionally ordered (111) terraces. All these voltammetric profiles are in good correlation with previous knowledge from Pt single crystal electrodes. As expected, all these states appear in all Pt nanoparticles but in a different extent, thus reflecting their specific surface structure. Both quasispherical Pt nanoparticles show similar surface site distributions than that observed with a polyoriented $\mathrm{Pt}$ surface. However, it is worth noting that the voltammetric response of the sample prepared using the general strategy proposed by J. Zhang and J. Fang ${ }^{[\mathbf{b b}]}$ but in absence of $\mathrm{W}(\mathrm{CO})_{6}$, shows a better definition of the adsorption states at 0.125 and $0.27 \mathrm{~V}$ as well as a relative higher weight of the contribution associated with the (100) surface domains. These features are related to the different size and level of agglomeration of the nanoparticles. On the other hand, the voltammetric response of the quasi-spherical sample prepared as detailed in reference ${ }^{[4 c]}$ is essentially similar to that found for small and quasi-spherical Pt nanoparticles prepared using other approaches including w/o microemulsion or

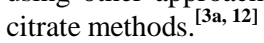

In the case of the cubic Pt nanoparticles, both synthetic approaches, in conjunction with the specific cleaning, allow the preparation of Pt nanoparticles with an evident (100) preferential orientation. Thus, in both samples, the signal at $0.35-0.37 \mathrm{~V}$ (related with the (100) terraces) is notably much more intense than the other one. Additionally, it is important to notice some particular differences between these similar samples. From the corresponding voltammetric responses, it can be observed that the contributions related to the (110) (at $0.125 \mathrm{~V}$ ) and (111) (at about $0.5 \mathrm{~V}$ ) are relatively more intense in the sample prepared with $\mathrm{Mn}_{2}(\mathrm{CO})_{10}$ as additive. ${ }^{[4 c]}$ This finding suggest a higher fraction of surface defects and/or some truncation with (111) planes in this sample thus resulting in a lower quality of the (100) surface structure of the nanoparticles. 


\section{Full Paper}

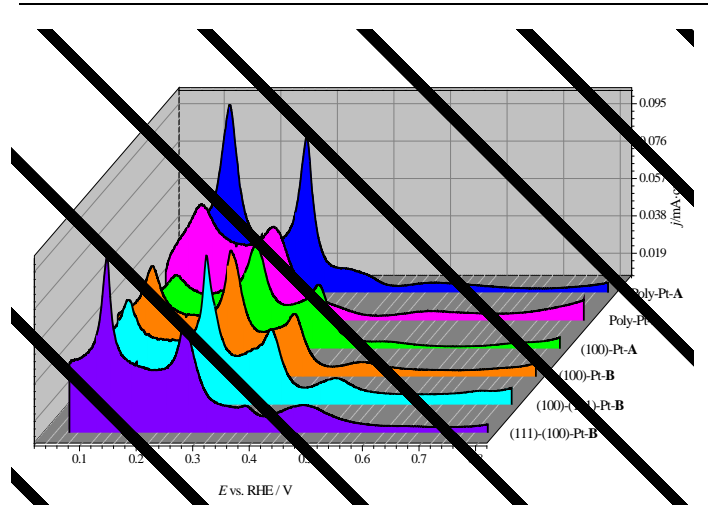

Fig. 6. Voltammetric profiles of shaped Pt nanoparticles synthesized by the OLA/OA method and washed with methanol+NaOH/Acetone. Test solution: $0.5 \mathrm{M} \mathrm{H} \mathrm{H}_{2} \mathrm{SO}_{4}$ sweep rate $=50 \mathrm{mV} \mathrm{s}^{-1}$.

On the other hand, the voltammetric profiles of the clean truncated cubic and octahedral Pt nanoparticles, both prepared using $\mathrm{Mn}_{2}(\mathrm{CO})_{10}$ as additive and benzyl ether as a co-solvent but at different reaction temperatures, suggest remarkable difference between their surface structures. At this respect, it is worth noting that such differences were hardly discernible during the TEM analysis, which highlights the benefits of this electrochemical analysis for the evaluation of the intrinsic surface structure at the nanoscale. In more detail, the response of the truncated cubic sample shows an important contribution of the (100) domains although less pronounced that that observed with the cubic sample. In addition, the contribution of the (111) surface domains is now much more predominant suggesting a higher degree of (111) truncation. This response is clearly different to that observed with the truncated octahedral nanoparticles for which the signal due to the (111) domains is now the predominant one. However, some contributions coming from (100) step and terraces are also observed but with a lower weight in the voltammetric response. Interestingly, as previously observed with preferentially oriented octahedral Pt nanoparticles, ${ }^{[3]}$ the contribution of the (110) sites is similar than those observed with (110) step sites on (111) terraces, coming from (111)x(111) junctions.

\subsubsection{Surface active sites quantification}

A more quantitative analysis of the relative amount of sites present on the surface of the nanoparticle can be also obtained by using other electrochemical surface probes sensitive to specific sites such as $\mathrm{Bi}$, Ge or Te adsorption

following the methodologies described in some of our previous contributions. ${ }^{[3,}$ 13] In particular, in this contribution, the percentage of the different ordered (100) and (111) domains was evaluated by using the adsorption of $\mathrm{Ge}$ and $\mathrm{Bi}$, respectively. Figures 7 and 8 show the representative responses of the different Pt nanoparticles obtained after each specific adsorption.

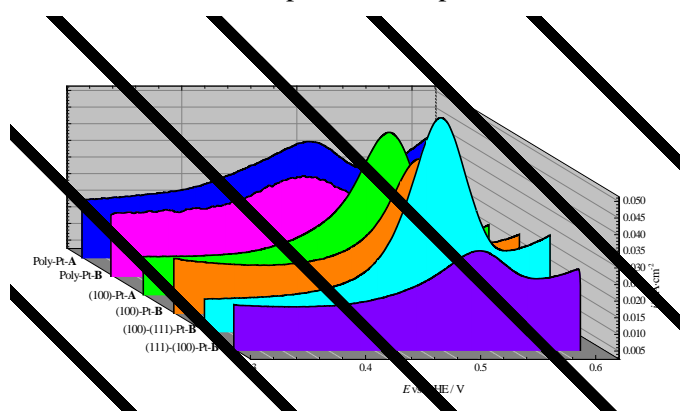

Fig. 7. Voltammetric profiles of the shaped Pt nanoparticles covered with reversible adsorbed germanium. Test solution: 0.5 $\mathrm{M} \mathrm{H}_{2} \mathrm{SO}_{4}$, sweep rate $=50 \mathrm{mV} \mathrm{s}^{-1}$.

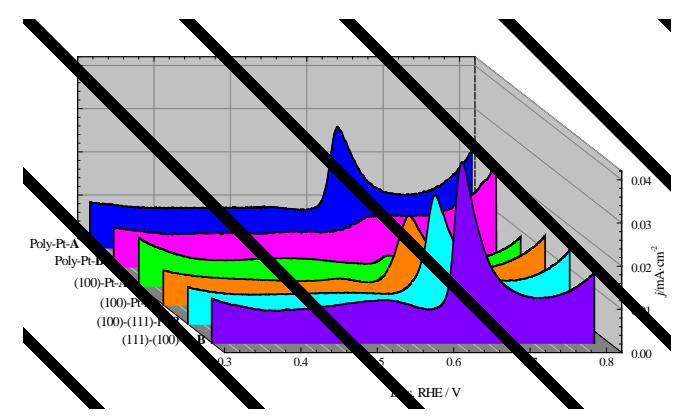

Fig. 8. Voltammetric profiles of the shaped Pt nanoparticles covered with irreversible adsorbed bismuth. Test solution: 0.5 $\mathrm{M} \mathrm{H}_{2} \mathrm{SO}_{4}$, sweep rate $=50 \mathrm{mV} \mathrm{s}^{-1}$.

Thus, from the charge involved in the oxidation process related to the presence of these irreversibly adsorbed adatoms and by using the relationships deduced for series of well-defined stepped surfaces, it is possible to quantify the fraction of (100) or (111) domains of each sample. Table 1 summarizes the results obtained for the different Pt nanoparticles.

Table 1. Fraction of the Pt(100) and Pt(111) ordered domains determined for the different nanoparticles. 


\begin{tabular}{cccc}
\hline Method & Sample & (100) sites, \% & (111) sites, \% \\
\hline \multirow{2}{*}{ Ref. ${ }^{[4 b]}$} & Poly-Pt-A & 14.2 & 10.5 \\
& (100)-Pt-A & 42.0 & 1.0 \\
& Poly-Pt-B & 14.1 & 1.0 \\
Ref. $^{[4 c]}$ & $(100)-P t-B$ & 27.0 & 8.8 \\
& (100)-(111)-Pt-B & 46.7 & 12.2 \\
& (111)-(100)-Pt-B & 14.3 & 18.3 \\
\hline
\end{tabular}

The results obtained are in good agreement with the qualitative analysis previously made from the voltammetric response of the samples in the so-called hydrogen region. As it can be extracted from these results, the adsorption of adatoms is a powerful tool to gain insight not only in the fraction of (100) and (111) sites, but also in the quality of these bidimensional domains. In this respect, note that both types of cubic Pt nanoparticles (both of comparable sizes) show similar TEM images but different percentage of (100) sites. This result indicates the presence of a bigger amount of defects on the surface of the nanoparticles (100)-Pt-B than in (100)-Pt-A, and those surface defects are not observable with the available microscopy techniques. Within all the samples studied in this work, the (100)(111)-Pt-B is the one with the highest percentage of (100) sites, pointing out that this sample must have wide and well-ordered (100) domains despite having a truncated structure. Note that all these information about the surface structure cannot been obtained by TEM analysis, which supplies enlightenment about the shape, size and agglomeration state of the nanoparticles, but not on the surface defects present on the different samples. It should be also mentioned that the baseline is a key element in the determination of the charge involved in the redox process of germanium, and in some cases, it may lead to a small overestimation of the values as compared with other techniques. ${ }^{11}$

\subsection{Electrocatalytic studies}

\subsubsection{CO stripping on shape-controlled Pt nanoparticles}

Previous studies using basal planes and Pt stepped electrodes have demonstrated that $\mathrm{CO}$ electro-oxidation is an extremely structure-sensitive reaction. ${ }^{[14]}$ Thus, CO has been widely used in Electrochemistry as a surface probe to identify the different symmetries and size of the domains present on the surface of the catalyst. These results can be correlated with those obtained for shapedcontrolled Pt nanoparticles both in acidic an alkaline media. ${ }^{[15]}$. Figure 9 displays the classical CO stripping experiments on the Pt nanoparticles under study. For each sample, the second CO stripping has been represented since it is well-known that the first CO electro-oxidation produces small changes due to removal of impurities and minimal surface reconstructions. ${ }^{[16]}$ Further CO adsorption/stripping processes do not affect the surface structure of the Pt nanoparticles.

The differences in the oxidation peaks observed in sulfuric acid for the different shaped-controlled $\mathrm{Pt}$ nanoparticles are due to the distinct sites where the CO electro-oxidation takes place. In this way, both types of quasi-spherical Pt nanoparticles present a CO stripping profile that reminds those recorded for polyoriented nanoparticles synthesized by the water in oil method. ${ }^{[17]}$ The slightly differences observed in the peak potentials are due to the different particle size and the agglomeration effect. As previously reported, ${ }^{[17]}$ the Pt nanoparticles with a preferentially (100) orientation present a characteristic sharp peak at ca. $0.75 \mathrm{~V}$ preceded by a shoulder at ca. $0.72 \mathrm{~V}$. It is worth to mention that, regardless of the method used to synthesize the nanoparticles, both the voltammetric profile and the $\mathrm{CO}$ stripping in the supporting electrolyte have the same aspect. In addition, shoulder at $0.72 \mathrm{~V}$ can be associated to the contribution of the defects present on (100) ordered domains in the sample, as its charge increases as the fraction of (100) ordered domains diminishes. Peak multiplicity in CO oxidation profiles appear when the Pt nanoparticles present a combination of both (111) and (100) domains.

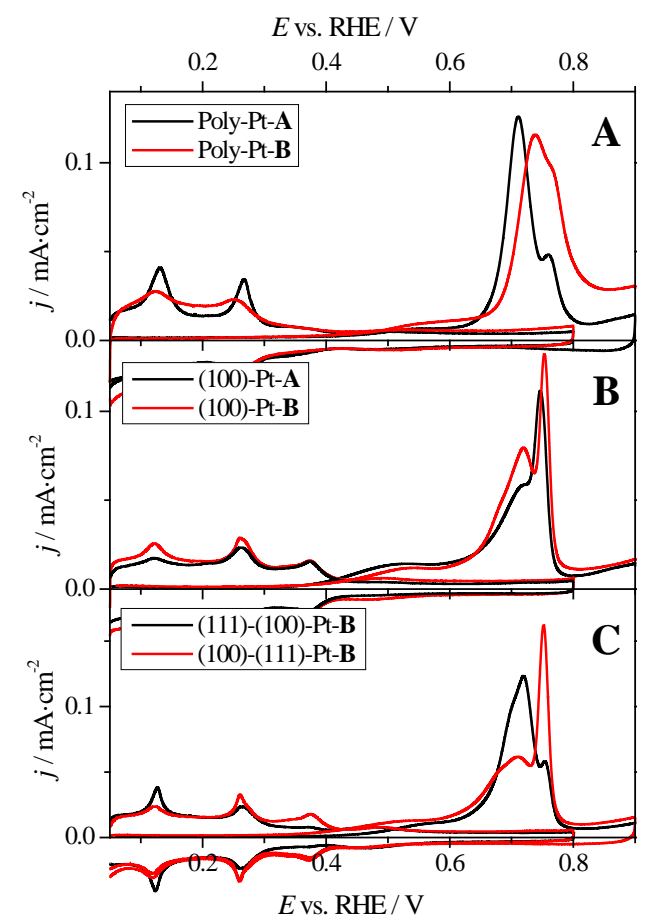


Fig. 9. CO stripping voltammograms of the different shaped $\mathrm{Pt}$ nanoparticles obtained by the methods described in (A) PolyPt, (B) (100)-Pt, (C) (111)-(100)-Pt and (100)-(111)-Pt. Test solution: $0.5 \mathrm{M} \mathrm{H}_{2} \mathrm{SO}_{4}$, sweep rate: $20 \mathrm{mV} \mathrm{s}^{-1}$.

\subsubsection{Ammonia oxidation and hydroquinone adsorption/desorption}

To further understand the electrocatalytic properties of these shaped Pt nanoparticles, some other structure sensitive reactions were also evaluated. As (100) structure sensitive reaction, we have used ammonia oxidation because is known to be extremely sensitive towards the Pt surface structure taking place almost exclusively on (100) sites. ${ }^{[18]}$ On the other hand, as (111) structure sensitive reaction, we will use the reductivedesorption oxidative-chemisorption of hydroquinonederived adlayers. ${ }^{[19]}$ As described in previous contributions, this process was reported to take place selectively from ordered $\mathrm{Pt}(111)$ domains present as terraces, being precluded at other available surface sites. Figure 10 reports the voltammetric profiles for both reactions for some of representative Pt nanoparticles. In the case of ammonia oxidation, peak currents follow the same order as the fraction of (100) sites determined by germanium adsorption, that is, the sample containing the fraction (100) ordered domains clearly shows the highest activity, corroborating the quantification of the sites made with the germanium probe.

Similarly, for the hydroquinone redox process, that sample showing a higher (111) contribution, provides a better activity towards this particular reaction, with the appearance of a well-defined peak at ca. $0.08 \mathrm{~V}$.
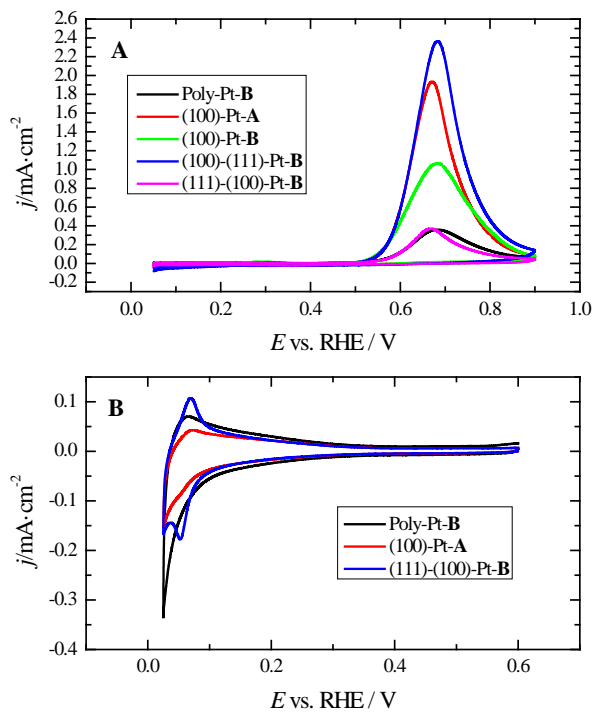

Fig. 10. (A) Voltammetric profiles for ammonia oxidation with platinum nanoparticles prepared by the OLA/OA method. Test solution, $0.2 \mathrm{M} \mathrm{NaOH}+0.1 \mathrm{M} \mathrm{NH}_{3}$; sweep rate, $10 \mathrm{mV} \mathrm{s}^{-1}$. (B) Steady voltammetric curves of these platinum nanoparticles in $2 \mathrm{mM} \mathrm{H}_{2} \mathrm{Q}+0.5 \mathrm{M} \mathrm{H}_{2} \mathrm{SO}_{4}$; sweep rate, $50 \mathrm{mV} \mathrm{s}^{-1}$.

\section{Conclusions}

A new decontamination protocol is reported for the elimination of the stabilizing agents remaining from the synthesis of shape-controlled Pt nanoparticles using the pair OLA/OA. In comparison with previous decontamination procedures, this methodology does not require an electrochemical step, which represents an important advance, in particular, if large amounts of sample is required for practical uses. In addition, this protocol minimizes the undesirable modification on the initial surface structure of the nanoparticles during the cleaning. The electrochemical characterization of the different Pt nanoparticles has been performed in sulfuric acid and the obtained results clearly demonstrate the effectiveness of this cleaning method by means of the presence of well-defined, sharp and reversible voltammetric peaks in the so-called hydrogen region. The results point out that, regardless of the synthesis method used to obtain the Pt nanocatalysts, similar voltammetric profiles must be acquired for nanoparticles with comparable morphology if the surface decontamination procedure has been successfully carried out. In addition, $\mathrm{Ge}$ and $\mathrm{Bi}$ adsorption, ammonia oxidation and hydroquinone adsorption/desorption reactions have been used to evaluate the amount and quality of the different sites present on the surface of the nanoparticles. The results will again demonstrate how the surface structure of the samples determines their electrocatalytic properties and that the presence of well-ordered domains and surface defects cannot be fully observed by the available microscopy techniques. Two samples with very similar shape and size, (100)-Pt-A and (100)-Pt-B, exhibit different electrocatalytic properties due to the presence of a larger number of surface defects in the (100)-Pt-B sample, which highlights the importance of a full electrochemical characterization of the nanoparticles to predict the electrochemical activity of them. Moreover, nanoparticles that have a cubic truncated shape presents the highest ratio of (100) ordered domains, because the surface planes present a very low amount of defects, in comparison with the other samples.

\section{Acknowledgements}




\section{Full Paper}

This work has been financially supported by the MICINN (Feder) of Spain and Generalitat Valencia through projects CTQ2013-44083-P PROMETEOII/2014/013, respectively. F.J.V.I. also thanks the European Social Funding.

\section{References}

a) M. T. M. Koper, Nanoscale 2011, 3, 2054-2073; b) J. SollaGullon, F. J. Vidal-Iglesias, J. M. Feliu, Annu. Rep. Prog. Chem., Sect. C 2011, 107, 263-297; c) S. C. S. L. Steven E. F. Kleijn, Marc T. M. Koper, Patrick R. Unwin, Angew. Chem. Int Edit. 2014, 53, 3558-3586; d) Z. Quan, Y. Wang, J. Fang, Acc. Chem. Res. 2013, 46, 191-202; e) H. Y. Jianbo Wu, Acc. Chem. Res. 2013, 46, 1848-1857; f) S. Y. Hongjun You, Bingjun Ding, Hong Yang, Chem. Soc. Rev. 2013, 42, 2880-2904; g) H. W. Nathan S. Porter, Zewei Quan, Jiye Fang, Acc. Chem. Res. 2013 46, 1867-1877.

[2] a) A. Björling, J. M. Feliu, J. Electroanal. Chem. 2011, 662, 17 24; b) A. Bjorling, E. Herrero, J. M. Feliu, J. Phys. Chem. C 2011, 115, 15509-15515; c) F. J. Vidal-Iglesias, J. Solla-Gullón, E. Herrero, V. Montiel, A. Aldaz, J. M. Feliu, Electrochem. Commun. 2011, 13, 502-505.

[3] a) J. Solla-Gullón, P. Rodríguez, E. Herrero, A. Aldaz, J. M. Feliu, Phys. Chem. Chem. Phys. 2008, 10, 1359-1373; b) F. J. Vidal-Iglesias, R. M. Aran-Ais, J. Solla-Gullon, E. Herrero, J. M. Feliu, ACS Catal. 2012, 2, 901-910. a) S. M. a. L. M. Liz-Marzán, Chem. Mater. 2013, 25, 14651476; b) J. Zhang, J. Fang, J. Am. Chem. Soc. 2009, 131, 1854318547; c) Y. Kang, J. B. Pyo, X. Ye, R. E. Diaz, T. R. Gordon, E. A. Stach, C. B. Murray, ACS Nano 2013, 7, 645-653; d) S. X. Sang-Il Choi, Minhua Shao, Ning Lu, Sandra Guerrero, Jonathan H. Odell, Jinho Park, Jinguo Wang, Moon J. Kim and Younan Xia, Chemsuschem 2014, 7, 1476-1483.

[5] D. Li, C. Wang, D. Tripkovic, S. Sun, N. M. Markovic, V. R. Stamenkovic, ACS Catal. 2012, 2, 1358-1362.
S. I. Choi, S. Xie, M. Shao, J. H. Odell, N. Lu, H. C. Peng, L. Protsailo, S. Guerrero, J. Park, X. Xia, J. Wang, M. J. Kim, Y. Xia, Nano Lett. 2013, 13, 3420-3425.

[7] H. Yang, J. Zhang, K. Sun, S. Zou, J. Fang, Angew. Chem. Int H. Yang, J. Zhang, K. Sun,
Edit. 2010, 49, 6848-6851.

[8] H. Yang, Y. Tang, S. Zou, Electrochem. Commun. 2014, 38 134-137.

[9] P. Rodríguez, E. Herrero, J. Solla-Gullón, F. J. Vidal-Iglesias, A. Aldaz, J. M. Feliu, Electrochim. Acta 2005, 50, 4308-4317.

[10] Y. Kang, M. Li, Y. Cai, M. Cargnello, R. E. Diaz, T. R. Gordon, N. L. Wieder, R. R. Adzic, R. J. Gorte, E. A. Stach, C. B. Murray, J. Am. Chem. Soc. 2013, 135, 2741-2747.

[11] a) J. Solla-Gullón, F. J. Vidal-Iglesias, P. Rodríguez, E. Herrero, J. M. Feliu, J. Clavilier, A. Aldaz, J. Phys. Chem. B 2004, 108, 13573-13575; b) Y. L. Vismadeb Mazumder, Shouheng Sun, Adv. Funct. Mater. 2010, 20, 1224-1234.

[12] J. Solla-Gullón, V. Montiel, A. Aldaz, J. Clavilier, J. Electrochem. Soc. 2003, 150, E104-E109.

[13] P. Rodríguez, E. Herrero, J. Solla-Gullón, E. J. Vidal-Iglesias, A. Aldaz, J. M. Feliu, Electrochim. Acta 2005, 50, 3111-3121. a) J. M. Leger, B. Beden, C. Lamy, S. Bilmes, J. Electroanal. Chem. 1984, 170, 305-317; b) N. P. Lebedeva, M. T. M. Koper, J. M. Feliu, R. A. van Santen, J. Phys. Chem. B 2002, 106, 12938-12947.

[15] a) Q. S. Chen, J. Solla-Gullon, S. G. Sun, J. M. Feliu, Electrochim. Acta 2010, 55, 7982-7994; b) M. J. S. Farias, F. J. Vidal-Iglesias, J. Solla-Gullón, E. Herrero, J. M. Feliu, J. Electroanal. Chem. 2014.

[16] A. López-Cudero, J. Solla-Gullón, E. Herrero, A. Aldaz, J. M. A. López-Cudero, J. Solla-Gullón, E. Herrero, A. A
Feliu, J. Electroanal. Chem. 2010, 644, 117-126.

[17] J. Solla-Gullón, F. J. Vidal-Iglesias, E. Herrero, J. M. Feliu, A Aldaz, Electrochem. Commun. 2006, 8, 189-194.

a) F. J. Vidal-Iglesias, J. Solla-Gullón, V. Montiel, J. M. Feliu, A. Aldaz, J. Phys. Chem. B 2005, 109, 12914-12919; b) F. J. Vidal-Iglesias, J. Solla-Gullón, P. Rodríguez, E. Herrero, V. Montiel, J. M. Feliu, A. Aldaz, Electrochem. Commun. 2004, 6 1080-1084.

[19] M. Rodriguez-Lopez, J. Solla-Gullon, E. Herrero, P. Tunon, J. M. Feliu, A. Aldaz, A. Carrasquillo, J. Am. Chem. Soc. 2010, 132, 2233-2242. 